\title{
eJRIEPS
}

Ejournal de la recherche sur l'intervention en éducation physique et sport

15 | 2008

Varia

\section{Alain Durey et les formations STAPS}

Jean-Pierre Cleuziou

OpenEdition

Journals

Édition électronique

URL : https://journals.openedition.org/ejrieps/5816

DOI : 10.4000/ejrieps.5816

ISSN : 2105-0821

Éditeur

ELLIADD

\section{Référence électronique}

Jean-Pierre Cleuziou, «Alain Durey et les formations STAPS », eJRIEPS [En ligne], 15 | 2008, mis en ligne le 01 juillet 2008, consulté le 03 mai 2021. URL : http://journals.openedition.org/ejrieps/5816 ; DOI : https://doi.org/10.4000/ejrieps.5816

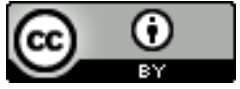

La revue eJRIEPS est mise à disposition selon les termes de la Creative Commons Attribution 4.0 International License. 
Alain Durey et les formations STAPS

Jean-Pierre Cleuziou

Quand Jean-François Robin m'a demandé de participer à l'hommage qui est rendu à Alain Durey au cours de ces journées, j'ai d'abord dit que d'autres personnes étaient mieux placées que moi pour exprimer ce qu'avait apporté Alain Durey aux STAPS et à la recherche en intervention.

Bien sûr, pendant quelques années, j'ai eu le privilège de travailler très près d'Alain à Orsay et de nouer avec lui des liens qui dépassent de beaucoup les relations professionnelles. Comme j'étais Directeur des Etudes STAPS à l'époque et que j'enseignais notamment l'athlétisme, les statistiques et l'informatique, les échanges avec Alain Durey étaient très fréquents et je dois dire que nous partagions sur presque tous les sujets les mêmes orientations et les mêmes démarches.

C'est de ce vécu que le GéDIAPS a souhaité que je témoigne en parlant du rôle d'Alain Durey dans les formations STAPS, tel que j'ai pu le connaître à Orsay. Je n'évoquerai donc qu'en pointillé son rôle dans l'orientation et le développement des recherches en didactique, laissant à d'autres les soins de situer son apport sur ce plan.

Lorsque Alain Durey est venu nous apporter sa collaboration en 1989 à Orsay, nous étions en pleine reconstruction du cursus STAPS et nous avions pour cela trois objectifs principaux :

nous dégager d'une tutelle inadaptée de l'UFR d'Orsay,

concevoir une formation des étudiants intégrant mieux enseignements scientifiques et connaissance approfondie des APS,

permettre à des étudiants ayant des formations secondaires très disparates de réussir en STAPS.

Je viens de dire que nous voulions nous dégager de la tutelle pédagogique de l'UFR d'Orsay et pour me faire comprendre, il faut que je précise que l'UFR d'Orsay était alors un centre universitaire de réputation mondiale orienté principalement vers les mathématiques, la physique, les sciences biologiques et l'informatique. II faut dire aussi qu'au début des années 80 la direction de l'université Paris-Sud et celle de l'UFR d'Orsay avaient voulu lutter contre l'échec massif des étudiants scientifiques en DEUG et avaient réformé le premier cycle universitaire en instaurant notamment un semestre d'orientation et des remises à niveau efficaces. 
Les responsables de cette réforme positive tenaient à englober les études STAPS dans cette action. Pour eux, en effet, on ne pouvait aborder valablement l'étude scientifique des activités physiques et sportives sans une formation conséquente en physique, en sciences biologiques et en mathématique. Ils pensaient aussi qu'il fallait instaurer des passerelles entre les études scientifiques et les études en STAPS.

Dans cette perspective, l'UFR et I'Université fournissaient aux STAPS des enseignants en mathématiques, en mécanique, en sciences biologiques mais aussi en psychologie puisque que le centre de psychologie cognitive du professeur Le Ny était implanté à Orsay.

Je dois dire que ces intentions généreuses, quoique fortement déductivistes, eurent des effets très négatifs sur les formations STAPS orcéennes. Lorsque je suis arrivé à Orsay en octobre 1985, j'ai été frappé par le nombre d'étudiants qui rendaient copie blanche en statistiques. D'une manière plus générale les étudiants contestaient l'orientation de leurs études dans de nombreuses disciplines. Beaucoup, qui avaient un bac littéraire ou technologique, peinaient pour comprendre des cours dont ils ne saisissaient pas, de plus, la finalité. Et à la fin de l'année, en juin 1986, deux étudiants seulement sur une cinquantaine obtinrent le CAPEPS.

II nous a fallu 4 à 5 ans d'efforts persévérants pour redresser une situation d'autant plus critique qu'avec une quinzaine d'enseignants du second degré et un seul Maître de Conférences, la division STAPS d'Orsay était une des structures STAPS les plus mal dotées de France.

C'est dans ce contexte qu'à la fin des années 80, Alain Durey est donc venu nous apporter son précieux concours, lorsqu'il intégra l'université Paris-Sud après le départ à Lyon de l'École Normale Supérieure de Saint-Cloud.

Nous avions négocié avec le département de physique d'Orsay, où il était affecté, la mise à disposition d'Alain Durey au profit des formations STAPS et pour nous ce fut une opération inestimable. Alain nous permit de transformer les contenus de formation en mécanique en DEUG 1ère année et de limiter fortement l'échec de nos étudiants. II contribua aussi à développer une formation à la recherche de la licence jusqu'aux formations doctorales. Enfin il contribua de façon déterminante à faire reconnaître en STAPS la nécessité et la spécificité des recherches en intervention.

Je ne peux développer ici chacun de ces points. Je me bornerai donc à quelques évocations personnelles. Quand Alain Durey accepta d'assurer la formation des 120 étudiants de DEUG STAPS 1ère année en mécanique, ce fut pour le Directeur des Etudes 
que j'étais un immense soulagement puis très vite un véritable bonheur. L'échec était massif chez nos étudiants et toute discussion avec l'enseignant que nous fournissait l'équipe de biomécanique d'Orsay était impossible.

Avec Alain, l'accord sur les orientations se fit rapidement. Compte tenu de l'hétérogénéité des formations secondaires de nos étudiants, il fallait partir des problèmes posés par les activités physiques pour construire des connaissances utiles pour la pratique personnelle de l'étudiant et pour l'analyse des prestations des autres pratiquants.

II fallait donc respecter une double logique : celle des différentes APS mais aussi celle de l'acquisition des connaissances en physique car leur nature et l'ordre dans lequel elles s'acquièrent ne sont pas bien sûr indifférents. C'est le sens de son ouvrage Physique pour les sciences du sport, fruit de ses quatre années d'enseignement à Orsay.

Alain aimait et connaissait les activités sportives, les étudiants trouvaient donc chez lui une écoute de qualité. II aimait aussi discuter avec les collègues pour se nourrir de leurs préoccupations et de leurs expériences. C'est ainsi que nous avons souvent échangé sur les enseignements que je réalisais en statistiques et qu'il réalisait en mécanique.

Ces discussions ont débouché sur diverses initiatives. Parmi elles, je citerai l'organisation de stages " natation et mécanique ». C'était une opération qui nous tenait à cœur car à ce moment il fallait faire un bond qualitatif dans la formation de nos étudiants tant en natation qu'en mécanique. La collaboration entre les deux Alain (Durey et Catteau) fut de grande qualité et nous aurions multiplié ce type de relation entre une APS et des " disciplines de service ", pour reprendre l'expression d'Alain Durey, si les contraintes sur les études STAPS ne s'étaient pas multipliées au cours des années 90 .

Comme on le voit, avec Alain Durey nous étions à cent lieues des conceptions de la formation que l'UFR d'Orsay avait expérimentées avec succès pour ses étudiants scientifiques mais qui avaient sécrété un échec massif dans les STAPS orcéennes. Nous avions le privilège de collaborer avec un spécialiste de la physique qui considérait sa discipline non pas comme une discipline fondamentale mais comme " une discipline de service dans le cursus STAPS à condition que les STAPS l'intègrent dans une perspective de production de connaissances finalisées par l'intervention » (Alain Durey, extrait d'une dédicace).

Après cette évocation du rôle d'Alain Durey en DEUG STAPS, qui pose bien sûr des problèmes actuels, je voudrais parler plus rapidement du concours qu'il nous a apporté dans la formation à la recherche en Licence, en Maîtrise et en DEA. Mais auparavant je voudrais adresser un grand merci au fidèle collaborateur d'Alain Durey, Philippe Varrin, pour sa disponibilité et ses compétences. Alain disait souvent que sans les compétences 
techniques de Philippe, il n'aurait pu réaliser bien des projets qui lui tenaient à cœur. Tous ceux qui ont travaillé de près avec Alain Durey savent ce qu'ils doivent aussi à Philippe pour la conduite et la réalisation finale de leurs travaux. Je tenais donc à les associer dans cette évocation.

Mais revenons à ces étudiants qui ont travaillé avec Alain et Philippe. À Orsay, les étudiants devaient réaliser en Licence et en Maîtrise un travail personnel important débouchant sur la rédaction d'un mémoire selon des normes scientifiques. Remarquons au passage qu'avec Alain Durey nous n'étions pas d'accord avec cette conception de l'initiation à la recherche et que nous préconisions plutôt la réplication d'expériences plus légères et plus diversifiées, permettant aux étudiants de se familiariser avec plusieurs démarches.

Mais ce n'est pas de cela que je veux surtout parler. Très vite un nombre assez important d'étudiants se tournèrent vers Alain Durey pour lui demander de les guider dans ce travail personnel. C'est que l'écoute d'Alain était d'une rare qualité et qu'il permettait aux étudiants de cheminer dans la voie qu'ils désiraient explorer tout en étant très rigoureux sur la démarche. Les étudiants qui s'adressaient à Alain savaient donc qu'ils travailleraient avec un enseignant compétent mais exigeant.

Je ne parlerai pas du rôle d'Alain Durey dans l'option APS du DEA de didactique que dirigeait Jean-Louis Martinand. Je pense que Daniel Bouthier évoquera ce rôle puisque c'est grandement à son initiative que cette option a pu se développer sous la responsabilité conjointe de Jacqueline Marsenach, d'Alain Durey et de lui-même. Jacqueline Marsenach, qui ne peut être parmi nous ce matin, m'a demandé de dire combien elle avait apprécié cette responsabilité conjointe, chacun développant ses orientations de recherche dans un esprit de complémentarité et de respect réciproque. Elle m'a demandé aussi de dire combien elle avait été sensible, durant ces années, à la culture, à la rigueur et à l'ouverture d'Alain Durey.

Ce sont ces compétences qui ont permis à Alain Durey de faire progresser en STAPS l'idée de la nécessité de recherches en intervention. On ne dira jamais assez ce qu'on lui doit sur ce plan car même les adversaires de la didactique et de la technologie des APS respectaient Alain pour ses incontestables compétences scientifiques.

Je dirai pour terminer que je le respectais aussi pour ses immenses qualités humaines. II est des êtres avec lesquels on se sent toujours bien. C'est cette sensation que je conserve d'Alain Durey parce qu'il était animé par des idéaux de justice et de progrès et que tout dans sa manière d'être incitait ceux qui le côtoyaient à partager ces idéaux avec lui. 